\title{
Proposição de um Modelo Matemático para Reduzir Custos no Processo de
}

\section{Psicodiagnóstico}

\author{
Maria Regina Martinez* \\ Universidade Federal de Alfenas - Unifal-MG, Alfenas, MG, Brasil \\ ORCID: https://orcid.org/0000-0001-6300-8980
}

\begin{abstract}
RESUMO
O psicodiagnóstico, a partir da aplicação de testes psicológicos validados cientificamente, sustenta o exame clínico convencional. Esses testes apresentam variações relacionadas à validade, amostras de estudo, evidências teóricas e empíricas que fundamentam sua pertinência, qualidade do material disponível, tempo necessário para sua aplicação e custos relacionados à aquisição do material de testagem e aos honorários profissionais necessários para concluir o processo de diagnóstico. Propõe-se, com um modelo matemático, um processo racional de psicodiagnóstico. Trata-se de um estudo quantitativo, descritivo-exploratório, baseado em dados de custo e tempo para aplicação de testes de inteligência. $O$ modelo matemático criado permitiu a elaboração de um protocolo de psicodiagnóstico de deficiência intelectual para aplicação em crianças e adultos considerando-se o melhor custo e efetividade.
\end{abstract}

Palavras-chave: administração em saúde, psicodiagnóstico, testes psicológicos.

\section{A Mathematical Model Proposal to Cost Reduction During Psychodiagnosis}

\begin{abstract}
The psychodiagnosis supports conventional clinical examination by psychological tests application. The available psychological tests present variations related to validity, samples, theoretical and empirical evidences, material's quality, time required for its application, and costs related to the acquisition of test material and the psychologist's earnings. This research proposes a rational process of psychodiagnosis. It is a quantitative, descriptive-exploratory study based on cost and time data for the application of standardized intelligence tests. We created a mathematical model that allowed the elaboration of a psychodiagnosis protocol for intelligence tests in children and adults considering the best cost-effectiveness.
\end{abstract}

Keywords: health care administration, psychodiagnosis, psychological testing. 


\section{Proposición de un Modelo Matemático para Reducir Costos en el Proceso}

\section{de Psicodiagnóstico}

\section{RESUMEN}

El psicodiagnóstico, a partir de la aplicación de pruebas psicológicas validadas científicamente, sostiene el examen clínico convencional. Estas pruebas presentan variaciones relacionadas con la validez, muestras de estudio, evidencias teóricas y empíricas que fundamentan su pertinencia, calidad del material disponible, tiempo necesario para su aplicación, y costos relacionados con la adquisición del material de prueba y los honorarios profesionales necesarios para concluir el proceso de diagnóstico. Se propone, con un modelo matemático, un proceso racional de psicodiagnóstico. Se trata de un estudio cuantitativo, descriptivo-exploratorio, basado en datos de costo y tiempo para aplicación de pruebas de inteligencia. El modelo matemático creado permitió la elaboración de un protocolo de psicodiagnóstico de deficiencia intelectual para aplicación en niños y adultos considerando el mejor costo y efectividad.

Palabras clave: administración en salud, psicodiagnóstico, pruebas psicológicas.

O psicodiagnóstico é uma avaliação psicológica, realizada privativamente por psicólogo, com propósitos diagnósticos, procurando avaliar forças e fraquezas no funcionamento psicológico do indivíduo, com um foco na existência ou não de psicopatologia. No psicodiagnóstico, há a utilização de testes e de outras estratégias para avaliar o examinando de forma sistemática, científica e orientada para a resolução de problemas (Cunha, 2009; Hutz, 2015).

A identificação da deficiência intelectual possibilita a compreensão do motivo pelo qual algumas crianças apresentam dificuldades de aprendizagem e permite o desenvolvimento de estratégias voltadas para as suas necessidades específicas (Silva, 2011). Na idade adulta, fundamenta a alocação profissional em cargos reservados legalmente para deficientes e oferece parâmetros para o dimensionamento das habilidades individuais (Ministério do Trabalho e Emprego [MTE], 2007). A categoria diagnóstica de Deficiência Intelectual referese à existência de uma limitação em qualquer área do funcionamento mental humano, abaixo da média esperada para a faixa etária. Nomeada pela Classificação Internacional das Doenças (Organização Mundial da Saúde [OMS], 1993) como Retardo Mental (CID-10: F70-F79), a Deficiência Intelectual pode ser considerada um transtorno neuropsiquiátrico, acometendo de 1 a $3 \%$ das crianças e adolescentes, sendo mais comum no sexo masculino (Marrus \& Hall, 2017). 
A Deficiência Intelectual tem início no desenvolvimento infantil e inclui déficits funcionais, tanto intelectuais quanto adaptativos, nos domínios conceitual, social e prático. A gravidade é definida com base no funcionamento adaptativo e não nos escores de Quociente de Inteligência (QI), uma vez que é o funcionamento adaptativo que determina o nível de apoio necessário. Os testes de inteligência e demais testes psicológicos podem ser aplicados no auxílio do diagnóstico e na identificação das funções cognitivas mais prejudicadas, permitindo o direcionamento das intervenções voltadas para o estímulo do indivíduo (DSM-5, 2013; Marrus \& Hall, 2017).

As concordâncias e discrepâncias obtidas entre os vários instrumentos do psicodiagnóstico são elementos preciosos para a construção do diagnóstico final. A avaliação diagnóstica deve se apoiar, ainda, sobre dados clínicos plurais construindo e reorganizando informações obtidas por procedimentos diversos no processo de investigação psicológica, dentre eles os dados obtidos na anamnese, nas entrevistas, nas provas cognitivas e nas provas projetivas. O psicólogo deve ser capaz de articular todos esses dados para construir um panorama total do paciente. Essa articulação não é um procedimento simples, dependendo de um conhecimento relativamente profundo de instrumentos variados (Silva, 2011).

A utilização desses instrumentos exige profissional qualificado, tempo disponível e material de testagem adequado, variáveis que determinarão o custo e a efetividade do psicodiagnóstico realizado. A criação do Sistema de Avaliação dos Testes Psicológicos (SATEPSI), em 2001, pelo Conselho Federal de Psicologia (CFP), para certificar a qualidade dos instrumentos de avaliação psicológica que podem ser usados profissionalmente, foi um grande avanço no suporte à prática profissional do psicólogo no psicodiagnóstico ao basear seu sistema de certificação em critérios internacionais de qualidade (Cardoso \& Silva-Filho, 2018).

De acordo com dados de 2015 do SATEPSI, os seguintes testes de inteligência receberam parecer favorável ao uso profissional como recursos auxiliares para o psicodiagnóstico: Bateria de Raciocínio Diferencial (BRD), Bateria TSP, BETA-III, Bateria de Provas de Raciocínio (BPR-5), G36, G-38, R-1, R-1 Forma B, Teste Não-Verbal de Inteligência para Crianças - R-2, Teste de Matrizes de Viena (WMT-2), Relógios - Teste NãoVerbal de Inteligência, Teste de Habilidade para o Trabalho Mental (HTM), Matrizes Progressivas de Raven, Teste Conciso de Raciocínio (TCR), Teste Não-Verbal de Raciocínio Infantil (TNVRI), Teste de Inteligência Não-Verbal - TONI-3, D70, Teste de Raciocínio Analógico Dedutivo (TRAD), Teste de Cubos, Teste de Inteligência (TI), Teste Equicultural de Inteligência (TEI), Teste de Inteligência Geral Não-Verbal (TIG-NV), Escala de 
Maturidade Mental Colúmbia (CMMS), Teste de Inteligência Verbal (TIV), V-47, SON-R 21/2-7[a], Escalas de Inteligência Wechsler para crianças e adultos - WISC-III, WISC-IV, WASI e WAIS-III (CFP, 2015). Cada um desses testes parte de uma concepção de inteligência, privilegiando determinados aspectos do funcionamento intelectual, e se utiliza de diferentes tipos de estímulos (verbais e/ou não verbais).

Para a seleção do teste a ser utilizado no psicodiagnóstico, conforme Barroso (2015), cabe ao profissional de psicologia ter clareza sobre os propósitos da avaliação, as características do examinando que são importantes para a execução do teste (a existência de deficiência visual ou auditiva, por exemplo), a descrição conceitual do constructo avaliado pelo teste, a relação de fontes de informação disponíveis para comparação dos resultados obtidos, a clareza e a qualidade das informações disponíveis no manual do teste, a adequação do teste quanto à validade em amostras compatíveis com o examinando, as evidências teóricas e empíricas que fundamentam a qualidade do teste na avaliação do constructo e a qualidade de todo o material disponível para aplicação do teste (estímulos, folhas de resposta, crivos e manual). Além disso, a apropriação do conteúdo do teste para os objetivos da avaliação psicológica, as características da amostra que participou da pesquisa de normatização do teste, a apropriação da normatização do teste para os propósitos da avaliação que será conduzida, o domínio por parte do profissional sobre o sistema de correção e interpretação dos resultados a serem obtidos no teste, a correspondência entre a interpretação possível dos resultados e a pergunta diagnóstica, a existência de publicações sobre o teste além do manual que trata da sua utilidade em diferentes contextos, a suficiência do conhecimento que o psicólogo tem sobre o teste para a sua seleção para uso na avaliação, e a adequação de como os resultados e interpretações serão relatados para as finalidades diagnósticas (Barroso, 2015).

A utilização de testes psicométricos comercialmente disponíveis é um grande desafio para o psicólogo, uma vez que na maioria dos manuais não existem parâmetros suficientes para sanar as dúvidas do profissional quanto à faixa etária indicada para a aplicação do teste, e seus parâmetros de padronização, validade, fidedignidade e normas (Reppold, Serafini, Gurgel, \& Kaiser, 2017; Reppold, Serafini, Ramires, \& Gurgel, 2017). As Escalas de Wechsler figuram entre as técnicas que se propõem a avaliar a inteligência como as mais bem elaboradas e tipificadas, sendo as de maior fidedignidade e validade repetidamente demonstrada. Ocupam um ponto referencial na psicologia contemporânea, demonstrando serem úteis para a medida do nível mental e para a interpretação clínica das possibilidades e limitações intelectuais do indivíduo. Sua principal relevância consiste em explorar uma amostra muito ampla de funções cognitivas, cuja relativa covariação e cujo progresso 
cronológico justificam a obtenção de uma medida geral da inteligência. As Escalas de Wechsler, reconhecidas mundialmente como padrão-ouro de testagem de inteligência, são utilizadas nas mais diversas áreas da prática psicológica, educacional e médica (Figueiredo, Vidal, \& Nascimento, 2015), embora sejam responsáveis por tornar o processo de psicodiagnóstico em deficiência intelectual demorado e oneroso, o que limita o seu acesso para uma parcela expressiva da população brasileira atendida em serviços de avaliação psicológica.

Poucos estudos avaliam estratégias que visem otimizar o processo de psicodiagnóstico, o que coloca em questão se há possibilidades para aumentar a sua eficiência. A visão de processo pode ser considerada uma forma para se realizar uma tarefa de maneira mais organizada, evitando-se desvios durante a execução da atividade e o consequente retrabalho. Quando se organiza uma tarefa em processo, é possível se estabelecer previamente os resultados que deverão ser obtidos e executá-la de uma maneira muito mais ágil e com menor custo, aumentando a efetividade (Kipper, Ellwanger, Jacobs, Nara, \& Frozza, 2011).

Por definição, processo é a introdução de insumos (entradas) em um ambiente formado por procedimentos, normas e regras que, ao processarem esses insumos, transformam-nos em resultados que serão enviados aos clientes do processo (saída). A gestão por processos permite que se agregue valor a partir do mapeamento e regulação de todos os processos envolvidos na geração do produto ou serviço fim (Kipper et al., 2011). A ferramenta mais utilizada para mapeamento e regulação de processos é o fluxograma.

Segundo Cunha (2009; p. 26), "psicodiagnóstico é um processo científico, limitado no tempo, que utiliza técnicas e testes psicológicos (input), em nível individual ou não, seja para entender problemas à luz de pressupostos teóricos, identificar e avaliar aspectos específicos, seja para classificar o caso e prever seu curso possível, comunicando os resultados (output), na base dos quais são propostas soluções, se for o caso". A gestão por processos em saúde prevê a utilização de protocolos clínicos no intuito de aumentar a eficiência da atenção prestada. No entanto, na área de avaliação psicológica poucos são os protocolos que têm sido desenvolvidos e utilizados no cotidiano do profissional de psicologia (Aznar-Blefari \& Padilha, 2015; Jardini, Ruiz, Ramalho, \& de Paula, 2015; Rodrigues Carreiro et al., 2014; Veltrone \& Mendes, 2011).

Nesse contexto, fica evidente que lidar com o psicodiagnóstico como um processo e introduzir conceitos relativos à gestão por processos pode trazer benefícios na relação de custo e benefício, com consequente aumento na eficiência no estabelecimento do diagnóstico. 
O estabelecimento do mapeamento e regulação do processo de psicodiagnóstico a partir da elaboração de fluxogramas pode subsidiar o desenvolvimento e utilização de protocolos clínicos norteadores da atividade do psicólogo, com o objetivo de orientar fluxos de procedimentos diagnósticos com o menor custo e o menor tempo, mantendo assegurada a assertividade da caracterização nosológica.

Para a determinação dos fluxos de procedimentos diagnósticos com o menor custo e o menor tempo é necessário se estabelecer indicadores objetivos que ofereçam apoio à tomada de decisão do profissional psicólogo. Nesse sentido, a utilização de modelos matemáticos capazes de relacionar as especificações técnicas dos testes psicológicos ao seu custo e tempo de aplicação, constitui-se uma estratégia poderosa para oferecer suporte à gestão por processos. O objetivo deste trabalho foi desenvolver um modelo matemático capaz de reduzir os custos relacionados à utilização de instrumentos psicométricos na avaliação do constructo inteligência, oferecendo suporte ao psicólogo na tomada de decisão relacionada ao desenvolvimento de protocolos eficientes no processo de psicodiagnóstico de deficiência intelectual em crianças e em adultos.

\section{Método}

A abordagem metodológica aplicada neste estudo é quantitativa, de natureza descritiva-exploratória, baseada no levantamento de dados de custo e tempo para aplicação de testes de inteligência com parecer favorável do Conselho Federal de Psicologia. Os dados foram coletados por meio do acesso às páginas da web do Sistema de Avaliação de Testes Psicológicos (SATEPSI) e sites que comercializam os referidos testes, entre setembro e novembro de 2015.

A amostra consistiu de 19 testes de inteligência direcionados para adultos e sete testes normatizados para crianças que continham dados disponíveis de custo e tempo de aplicação em sites voltados para a comercialização de testes psicológicos. Para todos os testes foram considerados os custos relativos a uma primeira aplicação, ou seja, os custos relacionados à aquisição de um kit completo do teste, composto por instrumentos de estímulos, folhas de resposta, crivos e manual. O tempo considerado para a aplicação dos testes foi aquele indicado nas especificações técnicas disponíveis nos seus sites de comercialização.

Os resultados são apresentados em gráficos de barras com os valores de custo e tempo expressos em percentual. Os dados obtidos foram analisados por estatística descritiva, sendo 
que os parâmetros de custo e tempo foram normalizados a partir do custo e tempo necessários para aplicação das Escalas de Inteligência Wechsler (WISC-IV e WAIS-III).

Os resultados da aplicação Escala Wechsler de Inteligência para Crianças - WISC IV em crianças com deficiência intelectual têm mostrado que o teste é uma medida de habilidade cognitiva muito útil a ser utilizada como apoio no diagnóstico clínico. Publicado em 2003, o WISC-IV é a escala mais usada para avaliar a inteligência de crianças cobrindo as idades de 6 anos a 16 anos, 11 meses e 30 dias. Fornece escores nas escalas verbal e de execução, bem como um QI de escala total. Inclui muitos tipos de tarefas, oportunizando a observação das dificuldades da criança e de suas habilidades (Costa, Azambuja, Portuguez, \& Costa, 2004).

A Escala Wechsler de Inteligência para Adultos (WAIS) foi elaborada com a finalidade de auxiliar na avaliação do funcionamento intelectual de adolescentes e adultos. É reconhecida como importante recurso diagnóstico para a identificação de diferentes habilidades cognitivas, possibilitando também a investigação do impacto de problemas emocionais, psiquiátricos e neurológicos no funcionamento cognitivo. Encontra-se em sua quarta edição, denominada WAIS-IV, embora a versão disponível para utilização no Brasil seja a sua terceira edição, o WAIS-III (Cunha, 2009; Figueiredo et al., 2015).

A partir da análise de parâmetros relativos a custo e tempo gastos para a aplicação de testes de inteligência, e a análise das especificações técnicas de cada teste analisado, desenvolveu-se uma modelagem matemática que fundamentou a proposta de um fluxograma norteador do processo de psicodiagnóstico em deficiência intelectual do adulto e da criança. $\mathrm{O}$ modelo matemático desenvolvido e apresentado, embora inicialmente criado para avaliar as características técnicas e de custo e tempo de aplicação de testes psicológicos de inteligência, pode ser utilizado para analisar instrumentos psicométricos desenvolvidos para a avaliação de outros constructos.

\section{Resultados}

A Figura 1 apresenta os testes de inteligência recomendados pelo Conselho Federal de Psicologia para adultos com seus respectivos preços e tempos de aplicação em comparação percentual com o WAIS-III. Todos os testes relacionados apresentam um custo percentual menor do que o WAIS-III e a maioria deles, segundo as informações do fornecedor, são aplicados utilizando-se menos tempo, com exceção do BPR-5. 


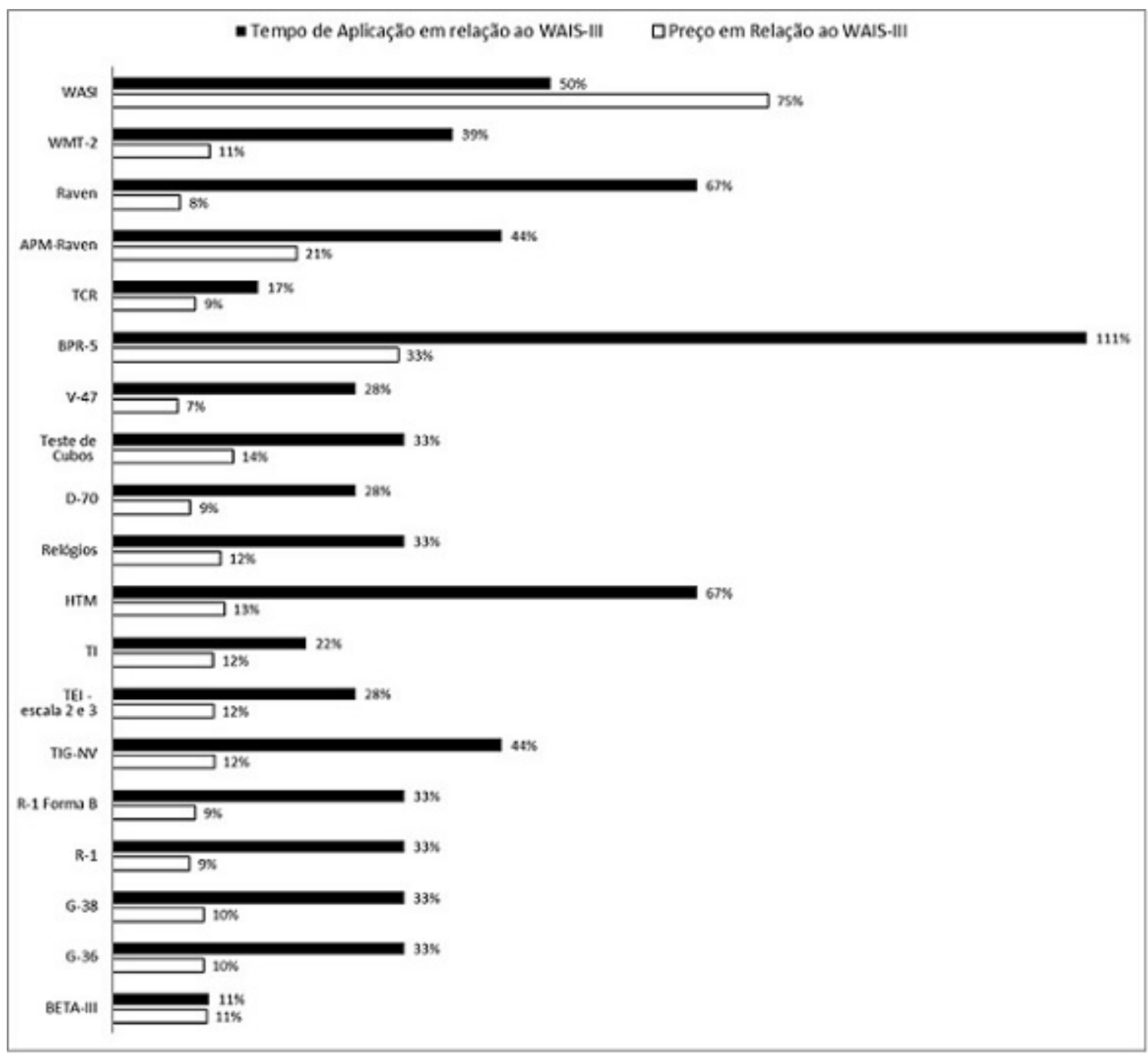

Figura 1. Testes de Inteligência normatizados para adultos e recomendados pelo Conselho Federal de Psicologia com seus respectivos preços e tempos de aplicação em relação percentual com o WAIS-III.

Nota. Segundo as informações dos fornecedores do WAIS-III, seu tempo de aplicação é de 90 minutos e o seu custo era de $\mathrm{R} \$ 1604,00$ no período de coleta de dados.

Com exceção do WASI, todos os demais testes apresentam um custo de aplicação que não chega a 40\% do custo do WAIS-III. Dos 19 testes avaliados, oito deles chegam a custar, no máximo, $10 \%$ do custo do WAIS-III. Dos testes considerados na amostragem, 16 deles são aplicados em metade do tempo ou menos do que o WAIS-III, sendo que 12 deles podem demorar até 33\% desse tempo em sua aplicação. Na Figura 2, são apresentados os testes de inteligência recomendados pelo Conselho Federal de Psicologia para aplicação em crianças com seus respectivos preços e tempos de aplicação em comparação percentual com o WISCIV. 


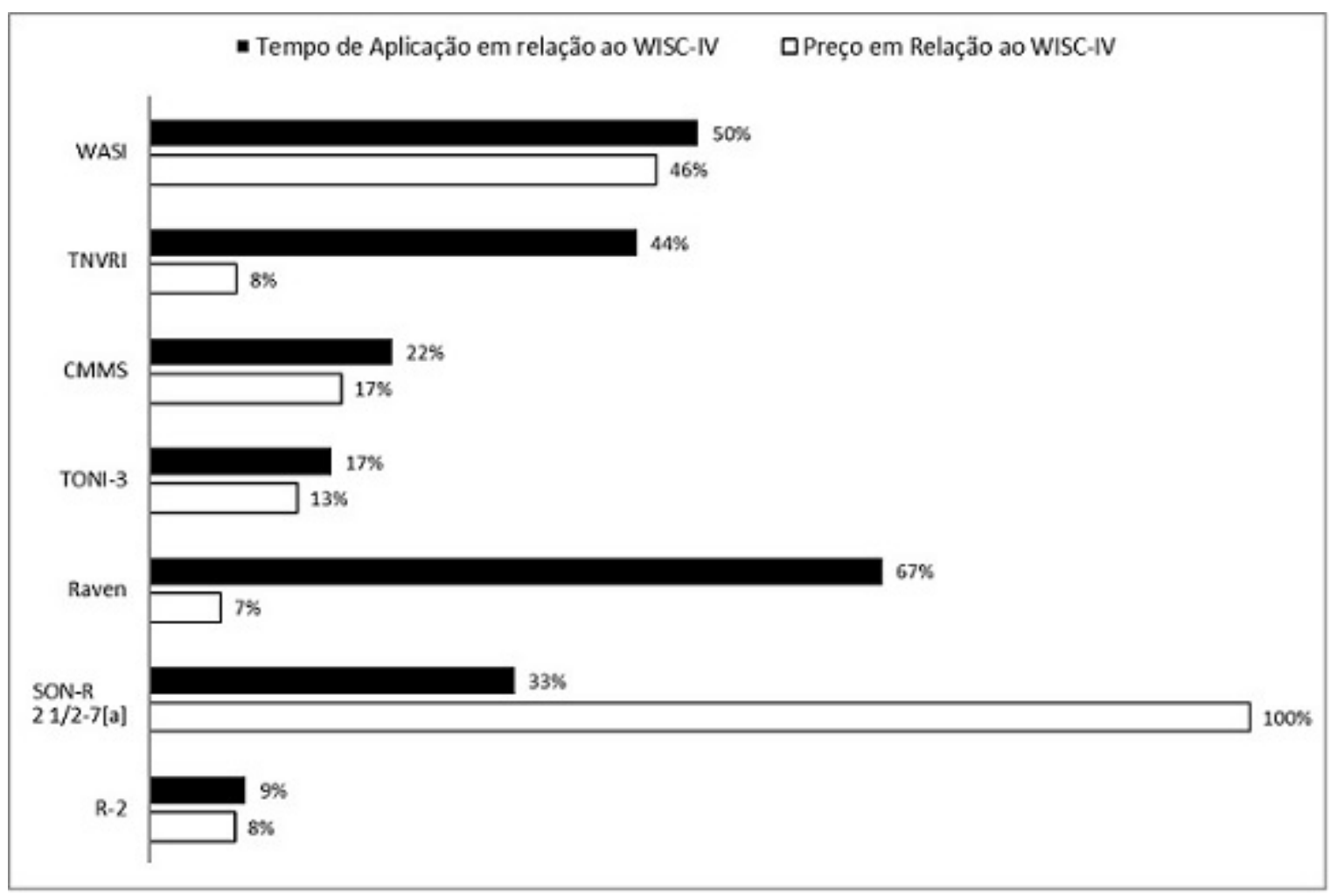

Figura 2. Testes de Inteligência normatizados para crianças e recomendados pelo Conselho Federal de Psicologia com seus respectivos preços e tempos de aplicação em relação percentual com o WISC-IV.

Nota. Segundo as informações dos fornecedores do WISC-IV, seu tempo de aplicação é de 90 minutos e o seu custo era de $\mathrm{R} \$ 2600,00$ no período de coleta de dados.

$\mathrm{Na}$ amostragem utilizada, o único teste que não tem um custo de aplicação inferior ao WISC-IV é o SON-R 21/2-7[a], sendo que a maioria dos demais não chega a custar nem metade do preço desses testes. Dos sete testes avaliados, três deles custam menos de $10 \%$ do WISC-IV. Todos os testes considerados na amostragem são aplicados na metade do tempo ou menos do necessário para aplicação do WISC-IV, sendo que quatro deles podem demorar até $33 \%$ desse tempo. A duração da aplicação dos testes de inteligência também participa do custo do psicodiagnóstico, desde que o psicólogo recebe por hora trabalhada. Portanto, dentre os parâmetros utilizados na modelagem matemática foi incluído o custo médio da hora para a realização de Avaliação Psicológica relacionada na tabela de referência de honorários dos Psicólogos, sugerida pelo Conselho Federal de Psicologia e disponível para consulta no seu site (CFP, 2017). A partir da análise dos resultados obtidos e valendo-se das premissas previstas em gestão de processos, propõe-se um fluxograma que pode nortear a elaboração de um protocolo clínico de psicodiagnóstico em deficiência intelectual (Figura 3). 


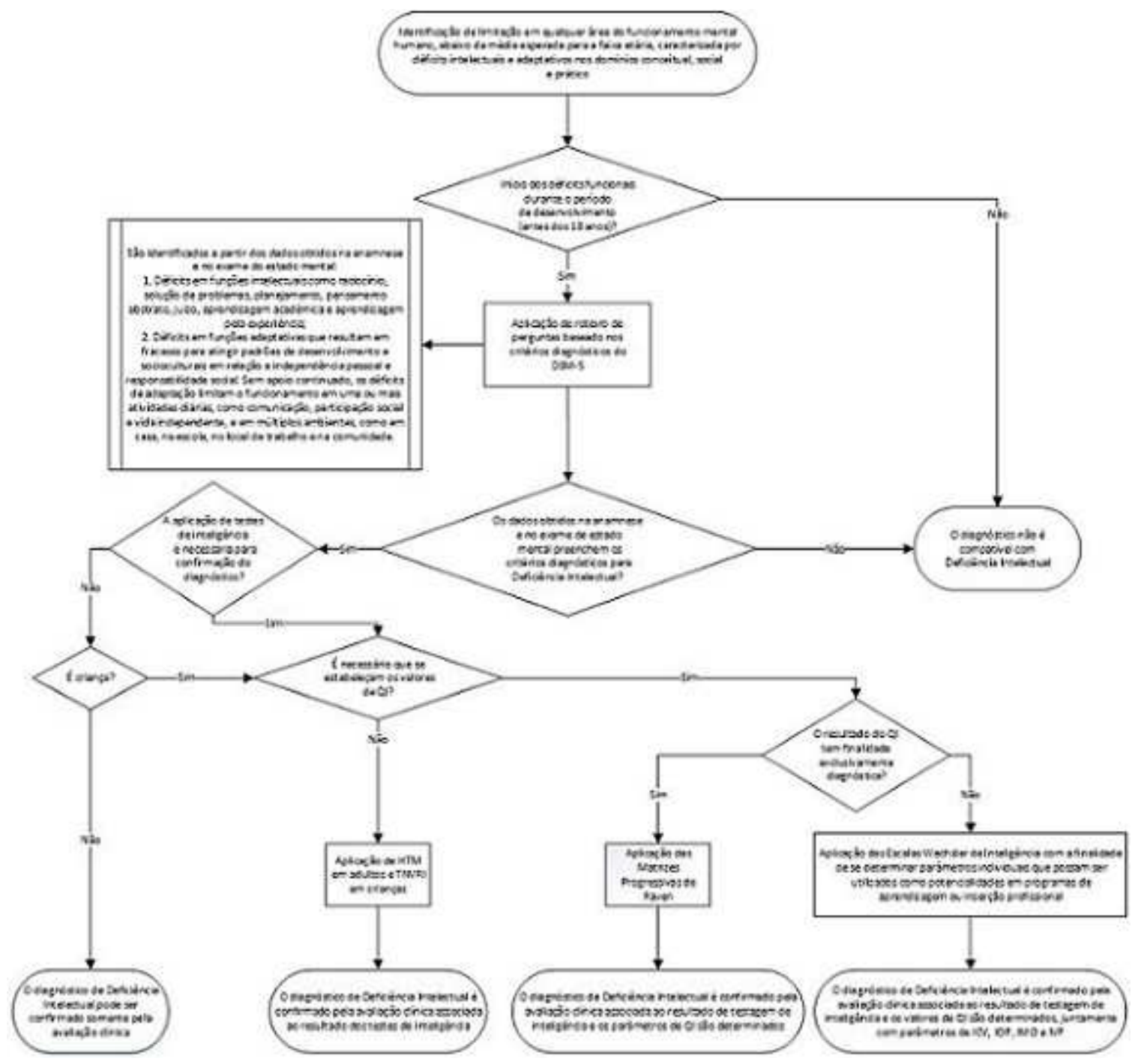

Figura 3. Proposta de fluxograma para subsidiar o processo de psicodiagnóstico em Deficiência Intelectual.

Os testes de inteligência inseridos no fluxograma foram selecionados seguindo-se os critérios de eficiência obtidos a partir da aplicação de um modelo matemático desenvolvido para oferecer apoio decisório ao psicólogo. A modelagem matemática considerou os seguintes parâmetros relacionados aos testes de inteligência utilizados: quantidade de testes de inteligência disponíveis no mercado e aprovados pelo Conselho Federal de Psicologia $(m)$, quantidade de características avaliadas pelos testes de inteligência disponíveis $(k)$, custo de aquisição de cada teste $\left(c t_{i}=\right.$ custo do teste $\left.i \forall i=1, \ldots, m\right)$, quantidade de horas a serem despendidas pelo profissional psicólogo para aplicação de cada teste $\left(q h t_{i}\right.$ do teste $i \forall i=1, \ldots$, $m$ ) e o custo médio da hora para avaliação psicológica de acordo com a sugestão do conselho da categoria $(c h)$.

Então, a partir dos resultados obtidos na Matriz 
$\delta_{c i} \forall c=1, \ldots, k ; i=1, \ldots, m$ onde

0 , se o teste $i$ não é capaz de identificar a característica $c$

1 , caso contrário.

Seleciona-se o teste de menor custo total:

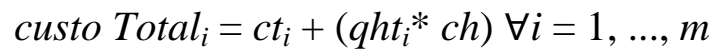

sujeito a

$\sum_{c=1}^{k} \delta_{c i}=k \forall i=1, \ldots, m$

Os testes selecionados atenderam aos parâmetros de validade, precisão e padronização previstos na literatura, critérios importantes para a garantia da sua eficácia como testes de inteligência (Cunha, 2009; Noronha, Freitas, \& Ottati, 2005). Para a utilização na prática clínica dessa modelagem matemática, o profissional de psicologia precisará contar com o apoio de algum software que permita a criação e edição de planilhas reguladas por fórmulas matemáticas, como o Microsoft Excel, dada à quantidade de características avaliadas pelos testes de inteligência disponíveis (representada pela letra $k$ na Equação 1) e demais dados que deverão ser relacionados. Uma vez realizada a modelagem do banco de dados compilando todos os testes disponíveis $(m)$, bem como seus custos e tempo para aplicação $\left(c t_{i}\right.$ e $q h t_{i}$, respectivamente), será necessário que o profissional correlacione esses resultados à escolha dos testes que realmente atendam às suas necessidades de avaliação, formalizadas matematicamente na Equação 1. À primeira vista, a formalização matemática pode parecer complexa ao psicólogo clínico, mas, uma vez que o banco de dados é elaborado e as fórmulas são inseridas em um software capaz de editar planilhas e realizar cálculos, o profissional poderá contar com um sistema bastante robusto de apoio decisório para a escolha de testes psicométricos.

Trabalhos futuros que se utilizem da formalização matemática aqui apresentada para a criação de aplicativos ou de planilhas de cálculo pré-definidas poderão encontrar um bom ambiente para o desenvolvimento de produtos que possam ser direcionados ao mercado voltado para a avaliação psicológica, sendo, inclusive, uma possibilidade interessante de serviço a ser oferecido pelas editoras de testes psicológicos. Considerando que, no adulto, graves déficits em funções intelectuais e funções adaptativas, com início na infância, sugerem a hipótese diagnóstica de Deficiência Intelectual após uma avaliação clínica sistematizada, se 
não há impressões diagnósticas sugestivas de outras morbidades que exijam um diagnóstico diferencial ou uma pretensão de seguimento terapêutico voltado para estimulação neurocognitiva, não há razão de expor esse indivíduo à aplicação de testes de inteligência envolvendo custos e tempo adicionais, além de um desgaste desnecessário por parte tanto do psicólogo examinador quanto do examinando para a elaboração de um diagnóstico definitivo. Portanto, sugere-se que, em situações em que os dados obtidos na anamnese e no exame de estado mental preencham os critérios diagnósticos do DSM-5 para Deficiência Intelectual e não há a necessidade de se excluir outras possibilidades diagnósticas, não sejam aplicados testes de inteligência.

$\mathrm{Na}$ criança, dada a importância de uma estimulação neurocognitiva sistematizada de início precoce a fim de restituir parte das perdas intelectuais e melhorar o prognóstico, sugerese que os testes de inteligência sempre acompanhem o processo de psicodiagnóstico, obtendose, assim, parâmetros para acompanhamento futuro do seu desenvolvimento cognitivo. Nas situações em que a aplicação de testes de inteligência se faz necessária, mas não é preciso que se estabeleçam parâmetros de QI, sugere-se que seja aplicado o Teste de Habilidades para o Trabalho Mental (HTM) no adulto e o Teste Não-Verbal de Raciocínio Infantil (TNVRI) para crianças.

O HTM, por meio de 48 questões divididas em três partes distintas, avalia os raciocínios lógico-verbal, lógico-numérico e lógico-abstrato, sendo capaz de fornecer subsídios para complementar os dados clínicos sugestivos do diagnóstico em deficiência intelectual (Santarosa, Wainstein, \& Prado, 1983). É um teste que custa expressivamente menos e tem um tempo de aplicação bem menor do que o WAIS-III (de 30 a 60 minutos dependendo dos anos de escolaridade do indivíduo testado).

O TNVRI tem por objetivo medir a capacidade de raciocinar por analogias e a capacidade de dedução (potencial intelectual) de crianças e jovens adolescentes. Tem se demonstrado útil para detectar deficiências na área de aptidão de raciocínio. É composto por 58 itens que avaliam dois fatores: raciocínio analógico abstrato (Fator 1) e raciocínio analógico concreto (Fator 2) (Cunha, 2009). Seu custo não atinge 10\% do investimento necessário para a aplicação do WISC-IV e o tempo de aplicação, embora variável, pode ser reduzido a 20 minutos.

Quando o estabelecimento dos parâmetros de QI é importante, mas tem finalidade exclusivamente diagnóstica, sugere-se que se associe à análise clínica a aplicação das Matrizes Progressivas de Raven - Escala Geral para adultos e Escala Especial (ou Matrizes Progressivas Coloridas) para crianças. Esse teste, em função do referencial teórico do qual 
deriva, avalia a inteligência medida pelo seu produto final, ou seja, pelos resultados, desconsiderando, dessa forma, os processos ou diferenças qualitativas que interferem nas respostas dos indivíduos (Bandeira, Alves, Giacomel, \& Lorenzatto, 2004). Embora as informações qualitativas individuais sejam perdidas e não seja um bom teste para o direcionamento de um programa sistematizado de estimulação neurocognitiva, seus custos e agilidade na aplicação quando comparado às Escalas Wechsler de Inteligência conferem vantagens quando a finalidade é somente de confirmação da impressão diagnóstica clínica.

Finalmente, se o objetivo do diagnóstico tem a intenção de estabelecer, seja no adulto ou na criança, algum tipo de conduta relacionada à estimulação neurocognitiva, programa de aprendizagem ou inserção profissional, é importante que se utilize no processo de psicodiagnóstico as Escalas Wechsler de Inteligência, com a finalidade de se obter os índices fatoriais de Compreensão Verbal (ICV), Organização Perceptual (IOP), Memória Operacional (IMO) e Velocidade de Processamento (IVP).

\section{Discussão}

Este estudo inicia um debate sobre a eficiência do psicodiagnóstico em deficiência intelectual em adultos e crianças no Brasil, ressaltando a variedade de testes de inteligência disponíveis no mercado com os mais diferentes custos e tempos de aplicação. Enquanto constructo, a inteligência tem interesse em ser medida a partir da hipótese de que pessoas altamente inteligentes possuem a habilidade de resolver problemas complexos em uma variedade de situações. O teste de QI por ele mesmo não é de inteligência, mas sobre como o comportamento inteligente se manifesta durante uma testagem padronizada. $\mathrm{O}$ comportamento inteligente existe associado a um contexto, portanto o ambiente determina como esse comportamento se manifesta, implicando que a testagem pode amplificar ou limitar a expressão da inteligência. Daí a importância do uso de testes fidedignos e extensamente testados para se traçar qualquer inferência sobre os níveis de inteligência individual (Holdnack \& Weiss, 2006).

Embora questionadas, as escalas de medição da inteligência ainda são amplamente usadas no processo de psicodiagnóstico da deficiência intelectual - inclusive por serem propostas como instrumentos diagnósticos nos manuais classificatórios como o DSM-5. Apesar da utilização de escalas de maturação e de comportamento para a avaliação do comprometimento no comportamento adaptativo nas dimensões conceituais, sociais e práticas, a obtenção dos valores de QI ainda é a informação considerada mais valiosa, 
determinando no CID-10 a classificação do indivíduo em seis categorias possíveis, a saber: F70 Retardo Mental Leve (QI na faixa de 50 a 69); F71 Retardo Mental Moderado (QI usualmente na faixa de 35 a 49); F72 Retardo Mental Grave (QI em média na faixa de 20 a 34); F73 Retardo Mental Profundo (QI abaixo de 20); F78 Outro Retardo Mental (nesta classificação a avaliação, por meio de procedimentos usuais, está prejudicada, como, por exemplo, diante da necessidade de se avaliar um indivíduo cego ou surdo; dessa forma, ele é classificado em Outro Retardo Mental) e F79 Retardo Mental Não Especificado (nesta categoria há evidência de retardo mental, mas as informações disponíveis são insuficientes para designar uma das categorias) (Bridi \& Baptista, 2014).

Embora pesquisas recentes questionem a viabilidade e adequação dos processos de testagem psicométrica da inteligência (Cristiano \& Toni, 2017; Kovacs \& Conway, 2019), a escassez de estudos que possam definir protocolos clínicos voltados para o psicodiagnóstico e intervenção neurocognitiva é um grande desafio do ponto de vista de assertividade diagnóstica e otimização de custos. Na falta de um mapeamento e regulação do processo de avaliação psicológica, por vezes são utilizados vários testes que não fornecem informações complementares e sim suplementares, além de se manterem algumas lacunas de compreensão acerca de alguns dados importantes para a definição diagnóstica.

A gestão por processos em organizações de saúde implica na implantação de protocolos clínicos que possam nortear a eficiência do estabelecimento de diagnóstico e tratamento em uma dada circunstância clínica e organizacional. No entanto, poucos estudos têm se preocupado em descrever a aplicação de protocolos no contexto do desenvolvimento de psicodiagnóstico, embora algumas tentativas tenham sido feitas principalmente no que se refere à avaliação de populações mais específicas, como idosos e crianças (Aznar-Blefari \& Padilha, 2015; Barbieri, 2010; Borba, 2012; Jardini et al., 2015; Rodrigues Carreiro et al., 2014; Veltrone \& Mendes, 2011).

Os protocolos são recomendações desenvolvidas sistematicamente para orientar as rotinas dos cuidados e das ações de gestão de um determinado serviço ou equipe. São elaborados a partir do conhecimento científico atual, respaldado em evidências empíricas aceitas pela comunidade científica e por profissionais experientes e especialistas em uma área. Orientam fluxos, condutas e procedimentos clínicos dos profissionais de saúde. São utilizados para auxiliar no manejo de um problema de saúde, numa circunstância clínica específica, fornecendo orientações concisas sobre testes diagnósticos e tratamentos. Constituem-se em importantes recursos para reduzir variações inapropriadas na prática clínica dos profissionais de saúde (Werneck, Faria, \& Campos, 2009). 
O trabalho clínico apresenta regularidades possíveis de serem identificadas e descritas, permitindo a elaboração de protocolos, embora deva ser considerada a combinação da padronização de procedimentos diagnósticos e terapêuticos às variações presentes em cada caso. Portanto, a definição e a aplicação de protocolos psicodiagnósticos está sempre relacionada a uma organização da atenção em saúde com autonomia profissional e responsabilidade clínica (Werneck et al., 2009).

Embora se reconheça que aspectos subjetivos do examinador e do examinando aparecem durante a testagem do constructo inteligência, podendo interferir na tomada de decisão clínica acerca da escolha dos testes psicométricos, considerando o custo da hora de trabalho do profissional psicólogo e os custos envolvidos na aplicação de testes psicológicos para a avaliação da inteligência, o processo de psicodiagnóstico deve ser conduzido com otimização econômica. Nesse sentido, a ponderação sobre quais testes devem ser utilizados durante a avaliação psicológica associada a esforços para o desenvolvimento de protocolos clínicos de psicodiagnóstico valorizam o trabalho do psicólogo e conferem maior eficiência e eficácia à sua atuação.

\section{Considerações finais}

A partir da análise de custos e de tempo de aplicação de testes de inteligência, propõem-se um modelo matemático capaz de oferecer apoio decisório na elaboração e implantação de protocolos clínicos voltados para o psicodiagnóstico de deficiência intelectual. Assim, assegura-se a aplicação eficiente de testes psicológicos como recursos diagnósticos complementares. A otimização dos custos e do tempo de aplicação dos testes de inteligência associada a um protocolo orientador do percurso de testagem permite um aumento do acesso da população aos serviços de psicodiagnóstico, acompanhado de um diagnóstico mais assertivo e uma orientação de conduta para o seguimento mais voltada para as necessidades individuais. Apesar de este estudo seja inovador no campo de psicodiagnóstico ao propor uma escolha de testes psicométricos a partir do ponto de vista da efetividade, estudos clínicos de caráter empírico que utilizem a modelagem matemática proposta deverão ser conduzidos para verificar se os testes de menor custo total conseguem atender também aos aspectos subjetivos do examinador e do examinando que aparecem durante a testagem do constructo inteligência. 


\section{Referências}

Aznar-Blefari, C., \& Padilha, M. da G. S. (2015). Capacitação para o uso do Protocolo NICHD em profissionais sul-brasileiros. Revista de Psicología, 24(1), 1-19. doi:10.5354/07190581.2015 .37198

Bandeira, D. R., Alves, I. C. B., Giacomel, A. E., \& Lorenzatto, L. (2004). Matrizes progressivas coloridas de Raven - escala especial: Normas para Porto Alegre, RS. Psicologia Em Estudo, 9(3), 479-486. doi:10.1590/S1413-73722004000300016

Barbieri, V. (2010). Psicodiagnóstico tradicional e interventivo: Confronto de paradigmas? Psicologia: Teoria e Pesquisa, 26(3), 505-513. doi:10.1590/S0102-37722010000300013

Barroso, S. M. (2015). Avaliação Psicológica. Petrópolis, RJ: Vozes.

Borba, E. E. (2012). O gerenciamento de projeto na construção e implantação de um protocolo de assistência na área da saúde para pessoas com deficiência intelectual. Revista de Ciências $\begin{array}{llll}\text { Gerenciais, } & 16(23), & \text { 107-126. } & \text { Recuperado }\end{array}$ https://revista.pgsskroton.com/index.php/rcger/article/download/2007/1908

Bridi, F. R. S., \& Baptista, C. R. (2014). Deficiência mental: O que dizem os manuais diagnósticos? Revista Educação Especial, 27(49), 499-512. doi:10.5902/1984686X13393

Cardoso, L. M., \& Silva-Filho, J. H. (2018). Satepsi e a qualidade técnica dos testes psicológicos no Brasil. Psicologia Ciência e Profissão, 38, 40-49. doi:10.1590/1982-3703000209112

Conselho Federal de Psicologia [CFP]. (2015). Sistema de avaliação de testes psicológicos. Brasília, DF: CFP. Recuperado de http://satepsi.cfp.org.br/listaTeste.cfm?status=

Conselho Federal de Psicologia [CFP]. (2017). Tabela de Honorários. Brasília, DF: CFP. Recuperado de http://site.cfp.org.br/servicos/tabela-de-honorarios/

Costa, D. I., Azambuja, L. S., Portuguez, M. W., \& Costa, J. C. (2004). Avaliação neuropsicológica da criança. Jornal de Pediatria, 80(2 Supl), 111-116. doi:10.1590/S002175572004000300014

Cristiano, L. B., \& Toni, P. M. (2017). Estrutura fatorial das Matrizes Progressivas Avançadas de Raven (Série I). Revista Neuropsicologia Latinoamericana, 9(3), 35-41. Recuperado de https://www.neuropsicolatina.org/index.php/Neuropsicologia_Latinoamericana/article/view/ 354

Cunha, J. A. (2009). Psicodiagnóstico - V (5a ed. rev.). Porto Alegre: Artmed.

DSM-5. (2013). Diagnostic and Statistical Manual of Mental Disordes (5a ed.). Arlington, VA: American Psychiatric Association. 
Figueiredo, V. L. M., Vidal, F. A. S., \& Nascimento, E. (2015). A quarta edição do teste WAIS. Avaliação Psicológica, 14(3), 413-416. Recuperado de http://pepsic.bvsalud.org/scielo.php?script=sci_arttext\&pid=S1677-04712015000300014

Holdnack, J. A., \& Weiss, L. G. (2006). WISC-IV integrated: Beyond the essentials. In L. G. Weiss, D. H. Saklofske, A. Prifitera, J. A. Holdnack, WISC-IV Advanced Clinical Interpretation (pp. 201-274). Cambridge, Massachusetts: Academic Press. doi:10.1016/B978-0120887637/50007-3

Hutz, C. S. (2015). O que é avaliação psicológica: Métodos, técnicas e testes. In C. S. Hutz, D. R. Bandeira, \& C. M. Trentini (Eds.), Psicometria (pp. 11-21). Porto Alegre: Artmed.

Jardini, R. S. R., Ruiz, L. S. R., Ramalho, W., \& de Paula, A. V. (2015). Protocolo Lince de investigação neurolinguística (PLIN): Instrumento lúdico para conhecer habilidades de leitura e escrita. Rev Psicopedagogia, 32(97), 49-60. Recuperado de http://pepsic.bvsalud.org/scielo.php?script=sci_arttext\&pid=S0103-84862015000100006

Kipper, L. M., Ellwanger, M. C., Jacobs, G., Nara, E. O. B., \& Frozza, R. (2011). Gestão por processos: Comparação e análise entre metodologias para implantação da gestão orientada a processos e seus principais conceitos. Tecno-Lógica, 15(2), 89-99. Recuperado de https://www.researchgate.net/publication/274064134_Gestao_por_processos_comparacao_ e_analise_entre_metodologias_para_implantacao_da_gestao_orientada_a_processos_e_seus _principais_conceitos

Kovacs, K., \& Conway, A. R. A. (2019). A unified cognitive/differential approach to human intelligence: Implications for IQ testing. Journal of Applied Research in Memory and Cognition. doi:10.1016/j.jarmac.2019.05.003

Marrus, N., \& Hall, L. (2017). Intellectual Disability and Language Disorder. Child and Adolescent Psychiatric Clinics of North America, 26(3), 539-554. doi:10.1016/j.chc.2017.03.001

Ministério do Trabalho e Emprego. (2007). A inclusão das pessoas com deficiência no mercado de trabalho (2a ed.). Brasília: SIT.

Noronha, A. P. P., Freitas, F. A., \& Ottati, F. (2005). Parâmetros psicométricos de testes psicológicos de inteligência. Interação em Psicologia, 6(2). doi:10.5380/psi.v6i2.3307

Organização Mundial da Saúde. (1993). Classificação de Transtornos Mentais e de Comportamento da CID-10: Descrições Clínicas e Diretrizes Diagnósticas. Porto Alegre: Artes Médicas.

Reppold, C. T., Serafini, A. J., Gurgel, L. G., \& Kaiser, V. (2017). Avaliação de aspectos cognitivos em adultos: Análise de manuais de instrumentos aprovados. Avaliação Psicológica, 16(2), 137-144. doi:10.15689/AP.2017.1602.01 
Reppold, C. T., Serafini, A. J., Ramires, D. A., \& Gurgel, L. G. (2017). Análise dos manuais psicológicos aprovados pelo SATEPSI para avaliação de crianças e adolescentes no Brasil. Avaliacao Psicologica, 16(1), 19-28. doi:10.15689/ap.2017.1601.03

Rodrigues Carreiro, L. R., Schwartzman, J. S., Nunes Cantiere, C., Ribeiro, A. F., Adorna da Silva, N., Fernandes Martin, M. A., ... Triguero Veloz Teixeira, M. C. (2014). Protocolo interdisciplinar de avaliação neuropsicológica, comportamental e clínica para crianças e adolescentes com queixas de desatenção e hiperatividade. Psicologia: Teoria e Prática, 16(3), 155-171. Recuperado de http://pepsic.bvsalud.org/scielo.php?script=sci_arttext\&pid=S1516-36872014000300012

Santarosa, L., Wainstein, O., \& Prado, Z. (1983). HTM - Teste de Habilidades para o Trabalho Mental. São Paulo: Vetor.

Silva, M. (2011). História dos testes psicológicos. São Paulo: Vetor.

Veltrone, A. A., \& Mendes, E. G. (2011). Descrição das propostas do Ministério da Educação na avaliação da deficiência intelectual. Paidéia, 21(50), 413-421. doi:10.1590/S0103$863 X 2011000300014$

Werneck, M. F., Faria, H. P., \& Campos, K. F. C. (2009). Protocolos de cuidado à saúde e de organização do serviço. Belo Horizonte: Nescon/UFMG, Coopmed.

\section{Endereço para correspondência}

\section{Maria Regina Martinez}

Universidade Federal de Alfenas

Escola de Enfermagem - Sala R201-O

Rua Gabriel Monteiro da Silva, 700, Centro, Alfenas - MG, Brasil. CEP 37130-001

Endereço eletrônico: martinez@unifal-mg.edu.br

Recebido em: 09/11/2018

Reformulado em: 25/11/2019

Aceito em: 24/12/2019

\section{Notas}

* Enfermeira, Psicóloga e Professora Associada da Universidade Federal de Alfenas junto aos cursos de Medicina e Enfermagem.

Agradecimento: A autora agradece ao Prof. Dr. Humberto César Brandão de Oliveira pelo apoio na discussão e elaboração do modelo matemático utilizado na pesquisa e a Pró-Reitoria de Extensão da Universidade Federal de 
Alfenas pelo apoio às atividades dos Projetos de Extensão com interface em Pesquisa Observatório de Gestão Hospitalar e Desenvolvimento Humano e Escuta Terapêutica, onde este trabalho foi desenvolvido.

Financiamento: Pró-Reitoria de Extensão da Universidade Federal de Alfenas-Unifal-MG.

Este artigo de revista Estudos e Pesquisas em Psicologia é licenciado sob uma Licença Creative Commons Atribuição-Não Comercial 3.0 Não Adaptada. 